\title{
Path Integral Monte Carlo Simulations of $\mathbf{H}_{2}$ Adsorbed to Lithium-Doped Benzene:
}

\section{A Model for Hydrogen Storage Materials}

Lachlan P. Lindoy, ${ }^{1}$ Stephen J. Kolmann, ${ }^{1}$ Jordan H. D'Arcy, ${ }^{1}$ Deborah L. Crittenden, ${ }^{2}$ and Meredith J. T. Jordan ${ }^{1, \text { a) }}$

1) School of Chemistry, The University of Sydney, Australia

2) Department of Chemistry, University of Canterbury, Christchurch, New Zealand

(Dated: 11 September 2015)

Finite temperature quantum and anharmonic effects are studied in $\mathrm{H}_{2}-\mathrm{Li}^{+}$-benzene, a model hydrogen storage material, using path integral Monte Carlo (PIMC) simulations on an interpolated potential energy surface (PES) refined over the eight intermolecular degrees of freedom based upon M05-2X/6-311+G(2df,p) density functional theory calculations. Rigidbody PIMC simulations are performed at temperatures ranging from $77 \mathrm{~K}$ to $150 \mathrm{~K}$, producing both quantum and classical probability density histograms describing the adsorbed $\mathrm{H}_{2}$. Quantum effects broaden the histograms with respect to their classical analogues and increase the expectation values of the radial and angular polar coordinates describing the location of the center-of-mass of the $\mathrm{H}_{2}$ molecule. The rigid-body PIMC simulations also provide estimates of the change in internal energy, $\Delta U_{\text {ads }}$, and enthalpy, $\Delta H_{\text {ads }}$, for $\mathrm{H}_{2}$ adsorption onto $\mathrm{Li}^{+}$-benzene, as a function of temperature. These estimates indicate that quantum effects are important even at room temperature and classical results should be interpreted with caution. Our results also show that anharmonicity is more important in the calculation of $U$ and $H$ than coupling - coupling between the intermolecular degrees of freedom becomes less important as temperature increases whereas anharmonicity becomes more important. The most anharmonic motions in $\mathrm{H}_{2}-\mathrm{Li}^{+}$-benzene are the "helicopter" and "ferris wheel" $\mathrm{H}_{2}$ rotations. Treating these motions as one-dimensional free and hindered rotors, respectively, provides simple corrections to standard harmonic oscillator, rigid rotor thermochemical expressions for internal energy and enthalpy that encapsulate the majority of the anharmonicity. At $150 \mathrm{~K}$, our best rigid-body PIMC estimates for $\Delta U_{\text {ads }}$ and $\Delta H_{\text {ads }}$ are $-13.3 \pm 0.1$ and $-14.5 \pm 0.1 \mathrm{~kJ} . \mathrm{mol}^{-1}$, respectively.

\footnotetext{
a)Electronic mail: m.jordan@chem.usyd.edu.au
} 


\section{INTRODUCTION}

Physisorptive hydrogen storage materials such as metal-organic frameworks ${ }^{1}$ (MOF) and carbon-based materials, such as graphene or carbon nanotubes, ${ }^{2}$ have been widely investigated over the last decade in an effort to find a hydrogen storage material that meets the United States Department of Energy (DOE) targets. ${ }^{3}$ MOF materials are amongst the most promising candidates due to their high surface areas and tunable pore sizes. For example, at cryogenic temperatures, MOF-177 can store in excess of $10 \mathrm{wt} \% \mathrm{H}_{2},{ }^{4-6}$ meeting the DOE gravimetric hydrogen storage target. ${ }^{3}$ For practical use, however, hydrogen must be stored at temperatures close to room temperature, where MOF materials store approximately $0.5 \mathrm{wt} \%$ $\mathrm{H}_{2} \cdot{ }^{4,7}$ The reduction in $\mathrm{H}_{2}$ storage capacity with temperature, for both MOF and carbonbased materials, is a consequence of low $\mathrm{H}_{2}$ binding enthalpies (typically $3-7 \mathrm{~kJ} . \mathrm{mol}^{-1}$ ). ${ }^{8,9}$ For room temperature $\mathrm{H}_{2}$ storage to meet DOE targets, the binding enthalpy of $\mathrm{H}_{2}$ to MOF materials must be increased to $15-45 \mathrm{~kJ} \mathrm{~mol}^{-1} \cdot{ }^{10,11}$

One strategy to improve the enthalpy of binding is the incorporation of bare metal sites. ${ }^{12,13}$ Lithium atoms, as dopants, are particularly attractive because Li is light. Importantly, transfer of the Li valence electron into the lowest unoccupied molecular orbital of

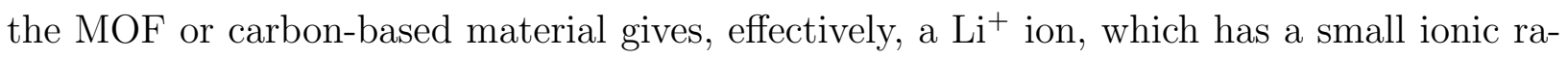
dius and a large charge density. In this case, $\mathrm{H}_{2}$ binding enthalpy is enhanced by favourable charge-quadrupole and charge-induced dipole interactions to the $\mathrm{Li}^{+} \cdot{ }^{11}$ Lithium is also appealing in terms of electronic structure calculations, since it has only three electrons.

The temperature at which $\mathrm{H}_{2}$ adsorption becomes thermodynamically favorable is sensitive to $\Delta H_{\text {ads }}$. Chavan et al. ${ }^{14}$ performed variable temperature infrared spectroscopic measurements on $\mathrm{H}_{2}$ adsorbed in MOF materials with bare metal sites and found that every $0.8 \mathrm{~kJ} \mathrm{~mol}^{-1}$ increase in $\Delta H_{\text {ads }}$ increases the $\mathrm{H}_{2}$ adsorption onset temperature by $10 \mathrm{~K}$. We therefore need at least $\mathrm{kJ} \mathrm{mol}^{-1}$ accuracy for calculated $\mathrm{H}_{2}$ adsorption enthalpies if we are to identify materials suitable for room temperature hydrogen storage.

To accurately calculate $\Delta H_{\text {ads }}$, we require accurate electronic energies, zero point energy and thermal energies. The most common way to correct electronic energies for temperature and zero point energy is to use harmonic thermochemical analysis. This assumes all vibrational frequencies are uncoupled and harmonic. Such an analysis is typically applied to covalently bound molecules where it can predict vibrational frequencies to within $5 \%$ 
of experimental frequencies. ${ }^{15}$ However, for a weakly-bound system such as $\mathrm{H}_{2}$ adsorbed to a porous material, harmonic thermochemical analysis may be much less accurate and a more rigorous treatment is required. We therefore investigated zero Kelvin properties of $\mathrm{H}_{2}$ adsorbed to $\mathrm{Li}^{+}$-benzene - a model system for Li-doped MOF and carbon-based hydrogen storage materials - using reduced-dimensional rigid-body diffusion Monte Carlo (RBDMC) simulations on modified Shepard interpolated potential energy surfaces (PESs) constructed at either the M05-2X/6-31+G(d,p) or M05-2X/6-311+G(2df,p) levels of theory. ${ }^{16}$ We had previously benchmarked the density functional calculations to more accurate coupled cluster calculations. ${ }^{16}$ Consistent with inelastic neutron scattering spectra of $\mathrm{H}_{2}$ adsorbed to a graphite intercalation compound, $\mathrm{KC}_{24},{ }^{17}$ we found that, at $0 \mathrm{~K}$, there was quantum delocalisation of the $\mathrm{H}_{2}$ molecule and, consistent with previous theory ${ }^{18}$ and experiment, ${ }^{19}$ the zero point energy difference upon binding is a significant fraction of the electronic binding energy, in this case $35 \%{ }^{20}$ We also found that the "helicopter" and "ferris wheel" motions of the adsorbed $\mathrm{H}_{2}$ molecule were significantly anharmonic and could be modelled as free and hindered rotors, respectively. Our best estimate of the $0 \mathrm{~K} \mathrm{H}_{2}$ adsorption enthalpy, $\Delta H_{\text {bind }}$ $(0 \mathrm{~K})$, was $-12.4 \mathrm{~kJ} \cdot \mathrm{mol}^{-1},{ }^{20}$ within $2 \mathrm{~kJ} \cdot \mathrm{mol}^{-1}$ of the experimentally measured $\Delta H_{\text {ads }}$ of $\mathrm{H}_{2}$ to bare metal sites. ${ }^{21}$

In this paper we extend our $0 \mathrm{~K}$ calculations to finite temperature; in the long term, room temperature $\mathrm{H}_{2}$ storage is required if $\mathrm{H}_{2}$ is to become a viable alternative to fossil fuels in vehicles. Moreover, the experimental characterisation of putative $\mathrm{H}_{2}$ storage materials typically takes place between 77 and $98 \mathrm{~K} .{ }^{22}$ Although quantum effects become less important as temperature increases, $\mathrm{H}_{2}$ is known to exhibit quantum behaviour at ambient temperature, ${ }^{17,19}$ and thus a quantum rather than classical formalism is required. Anharmonicity will become more important as temperature increases, especially for a weakly bound system such as $\mathrm{H}_{2}-\mathrm{Li}^{+}$-benzene and thus an accurate PES is also required. We have used Feynman's path integral formulation of quantum mechanics ${ }^{23,24}$ to compute thermodynamic quantities of interest in this system. Specifically, we perform rigid-body path integral Monte Carlo (RBPIMC) simulations on a M05-2X/6-311+G(2df,p) interpolated PES for $\mathrm{H}_{2}-\mathrm{Li}^{+}$-benzene at temperatures between 77 and $150 \mathrm{~K}$. The PES developed previously at $0 \mathrm{~K},{ }^{20}$ was extended, using the Grow algorithm, ${ }^{25}$ to model these higher temperatures. The estimates of $\Delta H_{\text {ads }}$ obtained from the RBPIMC simulations were compared to standard harmonic and approximate anharmonic estimates to examine the importance of quantum 
effects, anharmonicity and coupling as a function of temperature.

\section{METHODS}

\section{A. Electronic Structure Methods}

The electronic adsorption enthalpy of a $\mathrm{H}_{2}$ molecule to $\mathrm{Li}^{+}$-benzene is defined as:

$$
\Delta E_{\text {ads }}=E\left(\mathrm{H}_{2}-\mathrm{Li}^{+} \text {-benzene }\right)-E\left(\mathrm{Li}^{+} \text {-benzene }\right)-E\left(\mathrm{H}_{2}\right)
$$

where $E(\mathrm{~A})$ is the electronic energy of species $\mathrm{A}$. The $\mathrm{H}_{2}$ adsorption enthalpy $\Delta H_{\mathrm{ads}}$, at temperature, $T$, is:

$$
\Delta H_{\text {ads }}(T)=H\left(\mathrm{H}_{2}-\mathrm{Li}^{+} \text {-benzene, } T\right)-H\left(\mathrm{Li}^{+} \text {-benzene, } T\right)-H\left(\mathrm{H}_{2}, T\right)
$$

where $H(\mathrm{~A}, T)$ is the sum of $E(\mathrm{~A})$, the zero point energy of $\mathrm{A}$ and its internal energy, $U(\mathrm{~A}, T)$.

Following from our previous work, ${ }^{20}$ we have used the M05-2X ${ }^{26}$ density functional and the $6-311+\mathrm{G}(2 \mathrm{df}, \mathrm{p})^{27-29}$ basis set to describe the electronic energy of the $\mathrm{H}_{2}-\mathrm{Li}^{+}$-benzene complex. All electronic structure calculations were performed using the Gaussian03 $3^{30}$ and Gaussian09 $9^{31}$ program packages, with tight SCF convergence criteria, an ultrafine integration grid and with symmetry turned off.

\section{B. Potential Energy Surfaces}

A potential energy surface (PES) was constructed for $\mathrm{H}_{2}-\mathrm{Li}^{+}$-benzene at the M05-2X/6$311+\mathrm{G}(2 \mathrm{df}, \mathrm{p})$ level of theory using the modified Shepard interpolation of Collins and coworkers. ${ }^{32-34}$ In this method, the PES is expanded as a weighted second-order Taylor Series expansion about a set of $N_{\text {data }}$ data points and then iteratively improved, by adding additional data points, until calculated observables converge to within statistical error. The form of the modified Shepard interpolation allows the six-fold rotational symmetry of benzene and the two-fold symmetry of $\mathrm{H}_{2}$ to be included. The PES was "grown", using the Grow 2.2 program, and modifications thereof, ${ }^{25,32-35}$ sampling only intermolecular degrees of freedom. Thus, while the interpolated PES nominally describes the full (39-dimensional) 
system, only the intermolecular modes are accurately described. The method is summarised as follows:

1. The starting PES was the 100 symmetry-unique data point PES describing the ground state of $\mathrm{H}_{2}-\mathrm{Li}^{+}$-benzene, which we developed for our previous $0 \mathrm{~K}$ quantum diffusion Monte Carlo simulations. ${ }^{20}$ The 6 -fold rotational symmetry of the $\mathrm{Li}^{+}$-benzene complex was included by considering the reduced symmetry group to be comprised of the rotations about the $\mathrm{C}_{6}$ axis and the interchange of the $\mathrm{H}_{2}$ nuclei, that is, $|\mathrm{G}|=12$. The parameters used in the modified Shepard interpolation are identical to those used in Reference 20: a 1-part weight function with exponent $p=22$ and a weight cut-off for the neighbor list of $1.0 \times 10^{-5}$. These parameters and the 1-part weight function are defined in Reference 32.

2. RBPIMC simulations, using a rigid-body formulation of the path integral technique, as described below, were performed on the $\mathrm{H}_{2}-\mathrm{Li}^{+}$-benzene PES using the growing parameters described in Section II C.

3. The sampled RBPIMC configurations were used to identify up to three new PES points per iteration. These new data points were chosen using the method described in Reference 36.

4. A DFT frequency calculation was performed at each chosen configuration and the geometry, electronic energy, and first and second derivatives were added to the PES data set.

5. The internal energy and the probability density histograms of the reduced dimensional system were regularly monitored as a function of the number of symmetry-unique data points, $N_{\text {data }}$, using larger, more extensive, convergence simulations, as described in Section II C.

Steps 2 to 5 were repeated until convergence of the internal energy and nuclear probability densities - within the statistical errors of the simulations - was achieved. 


\section{Rigid Body Path Integral (PIMC) Simulations}

Although the path integral formulation of quantum mechanics has been previously described in many texts, ${ }^{23,24,37-39}$ its derivation is typically presented in a single dimension. We have rederived the primitive approximation path integral representation of the canonical partition function in three dimensions, using an internally consistent notation and in the form in which it has been implemented in this work. This derivation is given as Supplementary Material. ${ }^{40}$ We obtain the canonical partition function, $Q_{P}(\beta)$, for $N$ distinguishable particles in three dimensions, where the mass of particle $j$ is $m_{j}$, as:

$$
\begin{aligned}
Q_{P}(\beta)= & \prod_{j=1}^{N}\left(\frac{m_{j} P}{2 \pi \beta \hbar^{2}}\right)^{3 P / 2} \int \ldots \int \mathrm{d} \mathbf{R}^{(1)} \ldots \mathrm{d} \mathbf{R}^{(P)} \times \\
& \left.\exp \left\{-\beta\left[\sum_{t=1}^{P} \sum_{j=1}^{N} \frac{m_{j} P}{2 \beta^{2} \hbar^{2}}\left\|\mathbf{r}_{j}^{(t)}-\mathbf{r}_{j}^{(t+1)}\right\|^{2}+\frac{1}{P} \sum_{t=1}^{P} V\left(\mathbf{R}^{(t)}\right)\right]\right\}\right|_{\mathbf{R}^{(1)}=\mathbf{R}^{(P+1)}}
\end{aligned}
$$

where $\beta=1 / k_{B} T$, for $k_{B}$ the Boltzmann constant and $T$ the temperature. The application of Trotter's theorem ${ }^{41}$ corresponds to splitting the imaginary time propagator, $\hat{\rho}$, into $P$ short time propagators and $\mathbf{R}^{(t)}$ contains the coordinates of the system at the $t$ th imaginary time slice. The potential energy is solely a function of position, $\hat{V}=V(\hat{\mathbf{R}})$. The restriction $\mathbf{R}^{(1)}=\mathbf{R}^{(P+1)}$ in Equation (3) means that only closed paths (paths that start and finish at the same position) contribute to the partition function.

In this work, eight-dimensional rigid-body (RBPIMC) simulations were performed on $\mathrm{H}_{2}$ - $\mathrm{Li}^{+}$-benzene potential surfaces described in Section II B, where $\mathrm{H}_{2}$ was treated as a rigid rotor, with the $\mathrm{H}_{2}$ bond length fixed at its equilibrium value in $\mathrm{H}_{2}-\mathrm{Li}^{+}$-benzene, and benzene fixed in space. Whilst the PES is 39-dimensional and we can, in principle, perform ninedimensional PIMC simulations that include the $\mathrm{H}_{2}$ bond length coordinate, including the $\mathrm{H}_{2}$ vibration requires a significantly larger value for the Trotter number, $P$, because the characteristic timescales of vibrations differ significantly from rotations. We have explicitly frozen the $\mathrm{H}_{2}$ bond length because our primary focus is the intermolecular degrees of freedom associated with $\mathrm{H}_{2}$ adsorption. We further expect this to be a good approximation because we have previously shown, at $0 \mathrm{~K}$, that coupling between modes is less important than anharmonicity. ${ }^{20}$

The path integral representation of the canonical partition function, Equation (3), has been adapted for rigid bodies by separating the rotational and translational contributions 
to the rigid-body Hamiltonian, $\hat{H}_{\text {rigid }}$, that is,

$$
\hat{H}_{\text {rigid }}=\hat{K}_{\text {trans }}+\hat{K}_{\text {rot }}+\hat{V}
$$

where $\hat{K}_{\text {trans }}$ and $\hat{K}_{\text {rot }}$ are the translational and rotational kinetic energy operators, respectively. Here, for $N$ rigid rotors, the potential energy, $\mathrm{V}(\mathbf{R}, \boldsymbol{\Omega})$, is a function of $\mathbf{R}=$ $\left\{\mathbf{r}_{1}, \mathbf{r}_{2}, \ldots, \mathbf{r}_{N}\right\}$ and $\boldsymbol{\Omega}=\left\{\omega_{1}, \omega_{2}, \ldots, \omega_{N}\right\}$, the center-of-mass coordinates and the orientations of the $N$ rigid rotors. As derived in the Supplementary Material, ${ }^{40}$ estimators for the RBPIMC kinetic and potential energy are:

$$
\begin{gathered}
K_{\text {trans }}=\frac{3 N P}{2 \beta}-\left\langle\sum_{j=1}^{N} \sum_{t=1}^{P} \frac{m_{j} P}{2 \beta^{2} \hbar^{2}}\left\|\mathbf{R}_{j, t}-\mathbf{R}_{j, t+1}\right\|^{2}\right\rangle_{f_{P}} \\
K_{\text {rot }}=\left\langle\sum_{i=1}^{N} \sum_{t=1}^{P} \frac{B}{4 \pi \rho_{r o t, i}^{(t, t+1)}} \sum_{J=0}^{\infty}(2 J+1) J(J+1) P_{J}\left(\omega_{i}^{(t+1)} \cdot \omega_{i}^{(t)}\right) \exp \left(-\beta J(J+1) B_{i} / P\right)\right\rangle_{f_{P}} \\
V=\left\langle\frac{1}{P} \sum_{t=1}^{P} V\left(\mathbf{R}_{t}\right)\right\rangle_{f_{P}}
\end{gathered}
$$

where $\rho_{\mathrm{rot}, i}^{(t, t+1)}(\beta / P)$ is the rotational density matrix for rigid rotor $i, \omega_{i}^{(t)}$ and $\omega_{i}^{(t+1)}$ are the angular coordinate eigenfunctions and $f_{P}$ is the appropriate distribution function, Equation (S31). In this case we consider only the $\mathrm{H}_{2}$ molecule, so $N=1$ and $B=\hbar^{2} / 2 I$, for $I$ the moment of inertia, was calculated using the $\mathrm{H}_{2}$ bond length at the minimum energy structure of $\mathrm{H}_{2}-\mathrm{Li}^{+}$-benzene, that is, $0.7458 \AA$.

Evaluating $\rho_{\text {rot }}$ and $K_{\text {rot }}$ is computationally demanding and therefore, following Müser, ${ }^{42,43}$ Noya $^{44}$ and McBride, ${ }^{45}$ we pre-computed these on a one dimensional grid for each product of the number of beads and temperature, $P T$. During a simulation, required values of $\rho_{\text {rot }}$ and $K_{\text {rot }}$ were obtained from a spline interpolation of the grid. Additionally, evaluating $\rho_{\text {rot }}$ and $K_{\text {rot }}$ formally involves summing to infinite angular momentum, $J$. We truncated the sum over $J$ when it had converged to within $10^{-14}$.

The RBPIMC estimators were evaluated using Monte Carlo moves in the bead coordinates, $\mathcal{R}=\left\{\mathbf{R}^{(1)}, \ldots, \mathbf{R}^{(P)}, \boldsymbol{\Omega}^{(1)}, \ldots, \boldsymbol{\Omega}^{(P)}\right\}$. Each Monte Carlo step consisted of:

(i) Whole ring polymer displacements. Here moves corresponded to atom displacements, for the $\mathrm{Li}^{+}$ion, and center-of-mass displacements for the $\mathrm{H}_{2}$ molecule. On average two such moves were performed each Monte Carlo step. 
(ii) Staging moves. ${ }^{39,46}$ These moves were applied to the $\mathrm{Li}^{+}$ion and the center-of-mass of the $\mathrm{H}_{2}$. On average $2 P / M$ such moves were performed each Monte Carlo step, where $M$ is the staging length, as described in the Supplementary Material. ${ }^{40}$

(iii) Rigid body ring polymer rotation. In this case we consider only the rigid $\mathrm{H}_{2}$ molecule and consider random rotations of $\omega$ for all time slices. On average one such move was performed each Monte Carlo step.

(iv) Rigid body bead rotations. Here we consider random rotations, $\omega$, of the $\mathrm{H}_{2}$ molecule for one timeslice. On average $P$ such moves were performed each Monte Carlo step.

The probability of accepting a move from $\mathcal{R}_{\text {old }}$ to $\mathcal{R}_{\text {new }}$ was determined to ensure geometries were sampled from the distribution $f_{P}$, Equation (S31). Moves (i), (iii) and (iv) were accepted with a probability of $\min \left(1, \frac{f_{P}\left(\mathcal{R}_{\text {new }}\right)}{f_{P}\left(\mathcal{R}_{\text {old }}\right)}\right)$. The staging moves, moves (ii), were accepted with a probability of $\min (1, \exp [-\beta \Delta V])$. The Monte Carlo move parameters were chosen to ensure an acceptance ratio between 40 and $60 \%$. The $\mathrm{H}_{2}$ - $\mathrm{Li}^{+}$-benzene RBPIMC simulations used to iteratively grow the PES employed 2048 equilibration Monte Carlo steps and 16384 sampling steps. The purpose of these growing simulations was not to converge the internal energy but to sample the relevant configuration space in order to grow an appropriate PES. The final quantum RBPIMC results reported below were obtained on a PES defined using 300 symmetry unique data points. The convergence simulations employed 65536 equilibration steps and 524288 sampling steps. Details of convergence, as a function of simulation length, are provided in the Supplementary Material. ${ }^{40}$ Similar calculations were performed for $\mathrm{Li}^{+}$-benzene, using a potential energy surface constructed as a threedimensional spline interpolation of data from the 200 symmetry unique data point PES of reference 20. The isolated $\mathrm{H}_{2}$ molecule was treated as a free quantum rigid rotor with the bond length fixed at that of the M05-2X/6-311+G(2df,p) minimum energy geometry of $\mathrm{H}_{2^{-}}$ $\mathrm{Li}^{+}$-benzene, that is, $0.7458 \AA$. We have not considered relaxation of the $\mathrm{H}_{2}$ bondlength on binding to $\mathrm{Li}^{+}$-benzene.

Adsorption enthalpies, $\Delta H_{\text {ads }}$, are defined as the sum of the internal energy and a $p V$ term:

$$
H=U+p V
$$

where $p$ is the pressure and $V$ is the volume. Since we simulate one molecule only, we use 
the ideal gas approximation to estimate the $p V$ term, yielding:

$$
H=U+k_{B} T
$$

The $\mathrm{H}_{2}$ adsorption enthalpy becomes:

$$
\Delta H_{\mathrm{ads}}=\Delta U_{\mathrm{ads}}-k_{B} T
$$

Temperature dependent adsorption energies and enthalpies, $\Delta U_{\text {ads }}$ and $\Delta H_{\text {ads }}$, were calculated and compared to a zero Kelvin result, $\Delta H_{\text {ads }}(0 \mathrm{~K})$, obtained from RBDMC simulations as described in Reference 20. Probability densities and expectation values were determined for the $r$ and $\theta$ internal coordinates shown in Figure 1. The polar coordinate $r$ is the distance from the $\mathrm{Li}^{+}$ion to the $\mathrm{H}_{2}$ center-of-mass and $\theta$ is the angle subtended by $\mathbf{r}$ and the vector from the benzene center-of-mass to the $\mathrm{Li}^{+}$ion. We define an additional coordinate to describe the orientation of the $\mathrm{H}_{2}$ molecule: the ferris wheel angle between the $\mathrm{H}-\mathrm{H}$ bond and $\mathbf{r}$, which describes the orientation of the $\mathrm{H}_{2}$ molecule in a plane defined by the $\mathrm{Li}^{+}$ion and the two $\mathrm{H}$ atoms. The RBPIMC data was binned into histograms in $r, \theta$ and the ferris wheel angle. Note that the minimum energy $\mathrm{H}_{2}-\mathrm{Li}^{+}$-benzene geometry has the $\mathrm{Li}^{+}$ion on the benzene symmetry axis, as shown in Figure 1.

As described in the Supplementary Material, ${ }^{40}$ the internal energy, $U$, was converged, to within statistical error, with respect to the number of beads, $P$, at $77 \mathrm{~K}$ and $140 \mathrm{~K}$. This gave a ratio $\beta / P$ of approximately $0.0002 \mathrm{~K}^{-1}$ which was used to define $P$ at the other temperatures considered.

Classical simulations were also performed using $P=1$ beads. These $P=1$ results yield an internal energy where the rotational kinetic energy contribution is that of quantum free rotor, rather than a classical rotor. The classical results reported below correct for this. To ensure a similar number of configurations were sampled as the quantum simulations, the "classical" RBPIMC simulations employed 32 times the number of equilibration and sampling steps. Because the classical simulations were more sensitive to small variations in the PES, a larger, 350 symmetry unique data point PES was used for these calcualtions.

During the simulations, $\mathrm{H}_{2}$ may "desorb" from the $\mathrm{Li}^{+}$-benzene complex. We defined $\mathrm{H}_{2}$ as unbound if it were more than $7.5 \AA$ from the $\mathrm{Li}^{+}$ion. Errors in the RBPIMC internal energy were estimated using the blocking algorithm. ${ }^{47}$ 


\section{Rigid Body Harmonic Analysis}

A rigid-body harmonic vibrational analysis of $\mathrm{H}_{2}-\mathrm{Li}^{+}$-benzene was performed as described previously. ${ }^{20}$ The rigid-body harmonic normal modes are described here with reference to the coordinate system shown in Figure 1.

Harmonic frequency calculations were performed at the $\mathrm{H}_{2}-\mathrm{Li}^{+}$-benzene global minimum energy configuration within Gaussian03 with artificially high atomic masses $\left(1.0 \times 10^{18} \mathrm{amu}\right)$ for the benzene atoms. This results in nine non-zero harmonic normal modes:

- Two orthogonal $\mathrm{H}_{2}$-center-of-mass rocking motions, for example, in the $x z-$ and $y z-$ planes,

- Symmetric and antisymmetric stretches involving the $\mathrm{Li}^{+}-\mathrm{H}_{2}$-center-of-mass and $\mathrm{Li}^{+}$benzene-center-of-mass coordinates,

- Two orthogonal $\mathrm{Li}^{+}$"bends" parallel to the benzene plane,

- $\mathrm{H}_{2}$ "helicopter" rotation: $\mathrm{H}_{2}$ rotation about its center-of-mass in a plane perpendicular to $\mathbf{r}$,

- $\mathrm{H}_{2}$ "ferris wheel" rotation: $\mathrm{H}_{2}$ rotating around its center-of-mass in the plane defined bu the $\mathrm{Li}^{+}$ion and the two $\mathrm{H}_{2}$ hydrogen atoms,

- The $\mathrm{H}_{2}$ stretching vibration.

Thermochemical properties were calculated from the first 8 of these rigid-body normal modes, that is, excluding the $\mathrm{H}_{2}$ stretching vibration, using standard harmonic oscillator and rigid rotor expressions. ${ }^{48}$

\section{E. 1D DVR Anharmonic Energy Levels}

One-dimensional discrete variable representation (1D DVR) calculations were performed for the eight rigid-body normal modes. As described in Reference 20, normal mode displacement vectors were used to describe all but the helicopter and ferris wheel $\mathrm{H}_{2}$ rotations, which were approximated as rotational local modes, and the $\mathrm{H}_{2}$ rocking modes, which were modified to ensure the $\mathrm{H}_{2}$ bond length was maintained at its equilibrium value. 
The 1D DVR algorithm of Colbert and Miller ${ }^{49}$ was used to determine the one-dimensional energy levels in each of the rigid-body normal modes (or approximations thereof). The 0 to $2 \pi$ interval was used for the helicopter and ferris wheel $\mathrm{H}_{2}$ rotations and the $-\infty$ to $\infty$ interval was used for the remaining modes. The reduced masses for each normal mode were obtained from the rigid-body harmonic normal mode analysis performed in Gaussian03, with the reduced mass of $\mathrm{H}_{2}$ used to describe the helicopter and ferris wheel $\mathrm{H}_{2}$ rotations.

Potential energies required for the 1D DVR calculations were obtained from 1D spline interpolation of M05-2X/6-311+G(2df,p) electronic energies, evaluated as described in the Supplementary Material of Reference 20. 2000 DVR grid points were used for "stretching" vibrations and 1000 DVR grid points were used to describe bending and "rotational" motion. This ensured convergence to within $0.1 \mathrm{~cm}^{-1}$.

The 1D DVR partition functions were calculated via direct summation of the energy levels from $0 \mathrm{~K}$ to $300 \mathrm{~K}$ at $1 \mathrm{~K}$ intervals and were then used to calculate enthalpies for $\mathrm{H}_{2}$ adsorption to $\mathrm{Li}^{+}$-benzene using standard thermodynamic expressions. ${ }^{48}$ Analytic energy levels were obtained for the isolated $\mathrm{H}_{2}$ molecule which was treated as a free diatomic rigid rotor with the $\mathrm{H}_{2}$ bond length fixed at that of the M05-2X/6-311+G(2df,p) minimum energy geometry.

These calculations allow us to determine the relative importance of anharmonicity in individual normal modes and of coupling between modes in the thermodynamic quantities reported.

\section{RESULTS AND DISCUSSION}

\section{A. Probability Densities and Expectation Values}

Selected classical (dashed lines) and quantum mechanical (solid lines) probability density histograms in $r$ and $\theta$, obtained from the RBPIMC simulations are shown in Figure 2. The $r$ and $\theta$ coordinate definitions are as shown in Figure 1. For reference, $0 \mathrm{~K}$ probability density histograms obtained from a RBDMC simulation are also shown in Figure 2 as solid black lines. Errors in the RBDMC results, representing twice the standard error of the mean obtained from 10 independent RBDMC simulations, are indicated as grey shading around the solid, black lines in Figure 2. The standard error of the mean was also calculated 
from 16 independent RBPIMC simulations. These errors were within the thickness of the lines shown in Figure 2. All probability density histograms implicitly contain the $r^{2} d r$ or $\sin \theta d \theta$ volume elements. At the higher temperatures considered some of the RBPIMC simulations indicated desorption of the $\mathrm{H}_{2}$ molecule, defined as a $\mathrm{H}_{2}$ center-of-mass to $\mathrm{Li}^{+}$ distance exceeding $7.5 \AA$. Of the 16 independent quantum $(P \rightarrow \infty)$ RBPIMC simulations performed at each temperature, 10 simulations at $150 \mathrm{~K}$ reached a "desorbed" geometry, 6 at $140 \mathrm{~K}$ and 3 at $130 \mathrm{~K}$ and none at lower temperatures. Where "desorption" occurred, the results shown below were obtained from the remaining RBPIMC simulations. None of the classical $(P=1)$ simulations desorbed.

It can be seen from Figure 2(a) that the quantum $r$ probability density histograms (solid lines) are shifted to larger $r$ and are significantly broader than the classical $r$ probability densities (dashed lines). To quantify the difference between the classical and quantum results we computed expectation values, $\langle r\rangle$, and full-width-at-half-maximum (FWHM) of $r$ as a function of temperature. These results are shown in Table I. The RBPIMC errors in the expectation values shown in the table are in the last significant figure. Errors in the FWHM are also in the last significant figure and these have been calculated as the standard deviation in the FWHM from up to 16 independent RBPIMC simulations. At temperatures between $77 \mathrm{~K}$ and $150 \mathrm{~K}$, the quantum $\langle r\rangle$ values increase by approximately $0.04 \AA$ and the histograms broaden by $0.03 \AA$. The classical histograms show similar changes, $\langle r\rangle$ increases by approximately $0.05 \AA$ between $77 \mathrm{~K}$ and $150 \mathrm{~K}$ and the classical FWHM increases by 0.1 $\AA$. These changes are small, that is, there is little change in either the classical or quantum $r$ probability density histograms with temperature: the histograms shift very slightly to longer $r$ and become slightly broader as temperature increases from 77 to $150 \mathrm{~K}$.

The $\theta$ probability density histograms, shown in Figure 2(b), also exhibit temperature dependence, quantified in Table I. Between $77 \mathrm{~K}$ and $150 \mathrm{~K}$, the quantum $\langle\theta\rangle$ increases from approximately $26^{\circ}$ to approximately $30^{\circ}$, with the tails of the histograms extending to greater angles as the temperature increased. The quantum distributions also broaden with increasing temperature, the FWHMs increase from $32^{\circ}$ to $37^{\circ}$ over the range $77 \mathrm{~K}$ to $150 \mathrm{~K}$. The classical $\theta$ probability density histograms are peaked at lower $\theta$ than the corresponding quantum histograms, and are slightly narrower, as shown in Table I. The quantum and classical probability density histograms become increasingly similar as temperature increases.

We have previously considered the azimuthal, $\phi$, coordinate, shown in Figure 1 . At $0 \mathrm{~K}$ 
the RBDMC $\phi$ probability density histogram was constant, within error, indicating delocalisation of the $\mathrm{H}_{2}$ molecule in $\phi$. Although not shown, it is not surprising that RBPIMC $\phi$ probability density histograms also indicate that the $\mathrm{H}_{2}$ can be found, with equal probability, at any azimuthal angle. This is true for both classical and quantum simulations at all temperatures considered.

Probability density histograms were also generated for the "ferris wheel" coordinate, defined as the angle between the $\mathrm{Li}^{+}$ion, the center-of-mass of $\mathrm{H}_{2}$, and one of hydrogen atoms of $\mathrm{H}_{2}$ (see Figure 1). These are shown in Figure 3, with FWHMs for the probability density histograms given in Table II. At equilibrium, the $\mathrm{H}_{2}$ adopts a "T-shaped" orientation relative to the $\mathrm{Li}^{+}$ion, corresponding to a ferris wheel angle of $90^{\circ}$. Ferris wheel angles of $0^{\circ}$ and $180^{\circ}$ correspond to collinear alignment of the $\mathrm{H}_{2}$ and $\mathrm{Li}^{+}$with an energy approximately $26 \mathrm{~kJ} \mathrm{~mol}^{-1}$ above the global minimum. By symmetry, the expectation value of the ferris wheel coordinate is $90^{\circ}$ and all the ferris wheel probability density histograms are indeed symmetric about $90^{\circ}$. Figure 3 shows that, classically, $\mathrm{H}_{2}$ is found at ferris wheel angles between $60^{\circ}$ and $120^{\circ}$ at temperatures from $77 \mathrm{~K}$ to $150 \mathrm{~K}$, whereas the quantum probability density histograms show $\mathrm{H}_{2}$ is found at larger displacements from equilibrium, at ferris wheel angles between approximately $30^{\circ}$ and $150^{\circ}$. Increasing the temperature broadens the classical probability density histograms but has almost no effect on the quantum probability density histograms. This is consistent with the description of ferris wheel motion as a onedimensional hindered rotor. The energy barrier, approximately $26 \mathrm{~kJ} . \mathrm{mol}^{-1}$, is considerably larger than $k_{B} T$ and, even at $150 \mathrm{~K}$, only the lowest energy quantum state is significantly populated. Notably, this barrier is also considerably larger than the $\mathrm{H}_{2}$ desorption energy.

\section{B. Thermodynamics}

\section{Internal Energies, $\Delta U_{a d s}$}

Figure 4 shows various estimates of the change in internal energy, $\Delta U_{\text {ads }}$, of the $\mathrm{H}_{2}-\mathrm{Li}^{+}-$ benzene system upon adsorption of $\mathrm{H}_{2}$, as a function of temperature. Our best estimates of $\Delta U_{\mathrm{ads}}$, the quantum RBDMC and RBPIMC results, are indicated by the black circles in Figure 4. The RBDMC results shown in Figure 4 are slightly different to those reported in Reference 20. In our previous work the benzene molecule was treated as a rigid rotor 
whereas here it is held rigid. Classical RBPIMC estimates of $\Delta U_{\text {ads }}$ are indicated as black squares in Figure 4.

The blue solid line in Figure 4 above the RBDMC and RBPIMC estimates represents the "standard" harmonic oscillator, rigid rotor thermochemical estimate of $\Delta U_{\text {ads }}{ }^{48}$ calculated from the harmonic ZPE and vibrational frequencies of the minimum energy configuration. This estimate gives smaller magnitudes for $\Delta U_{\text {ads }}$, with respect to the RBDMC and RBPIMC results, at all temperatures considered. The difference in magnitude is between 1.5 and $1.8 \mathrm{~kJ} . m o l^{-1}$ between 77 and $150 \mathrm{~K}$ with the discrepancy increasing with increasing temperature. This suggests that the importance of anharmonic contributions to $\Delta U_{\text {ads }}$ increases with temperature. As a consequence, one-dimensional anharmonic corrections to $\Delta U_{\text {ads }}$ are also shown in Figure 4. If all eight intermolecular degrees of freedom in the $\mathrm{H}_{2}$ - $\mathrm{Li}^{+}$-benzene system are considered to be one-dimensional anharmonic oscillators, then anharmonic estimates for $\Delta U_{\text {ads }}$ can be calculated explicitly from energy levels determined from the one-dimensional discrete variable representation (1D DVR) calculations described in Section IIE. The 1D DVR estimate of $\Delta U_{\text {ads }}$ is shown as the dashed, dark green line in Figure 4. This result is in better agreement with the RBDMC and RBPIMC results than the standard harmonic oscillator, rigid rotor estimate but it also underestimates the magnitude of $\Delta U_{\mathrm{ads}}$. The difference between the 1D DVR estimates and the RBDMC and RBPIMC results indicates the importance of coupling in the $\mathrm{H}_{2}-\mathrm{Li}^{+}$-benzene system, viz. approximately $1.1 \mathrm{~kJ} \cdot \mathrm{mol}^{-1}$ at $0 \mathrm{~K}$, which decreases to approximately $0.7 \mathrm{~kJ} \cdot \mathrm{mol}^{-1}$ at $150 \mathrm{~K}$. Coupling between the intermolecular degrees of freedom becomes less important in $\Delta U_{\text {ads }}$ as the temperature increases, whilst anharmonicity becomes more important.

Classical approximations are only valid at high temperature, or more generally, where quantum effects are negligible. For the purposes of this work, they provides a way of quantifying the importance of quantum effects at finite temperatures. The discrepancy between quantum RBDMC/RBPIMC and classical RBPIMC results is, as expected, largest at $0 \mathrm{~K}$. This reduces to an approximately $3.3 \mathrm{~kJ} . \mathrm{mol}^{-1}$ difference at $150 \mathrm{~K}$, or $17 \%$ of the $\mathrm{H}_{2}-\mathrm{Li}^{+}$benzene electronic binding energy. At room temperature there is still more than a $2 \mathrm{~kJ} . \mathrm{mol}^{-1}$ difference between quantum and classical estimates of $\Delta U_{\text {ads }}$. These differences highlight the importance of including quantum effects when simulating $\mathrm{H}_{2}$ adsorption to $\mathrm{Li}^{+}$-benzene, and suggest that, even at room temperature, classical simulations will significantly overestimate the strength of the $\mathrm{H}_{2}$ binding interaction. 
The simplest classical estimate for $\Delta U_{\text {ads }}$ is obtained from the equipartition principle of statistical mechanics and is shown as the light green alternating long- and short-dashed line. Here we have assumed that the only contribution to $\Delta U_{\text {ads }}$ is the loss of three translational and one rotational (the ferris wheel rotation) degree of freedom of the $\mathrm{H}_{2}$ molecule as it binds to $\mathrm{Li}^{+}$-benzene. Thus the equipartition theorem estimate of $\Delta U_{\text {ads }}$ is a straight line of slope $2 k_{B} T$. This simple estimate is in excellent agreement with the classical RBPIMC results over the temperature range considered.

\section{Simple Methods to Compute $\Delta U_{a d s}$}

Given it is necessary to include quantum effects to accurately determine $\Delta U_{\text {ads }}$, simple, fast and accurate corrections to standard harmonic estimates are both useful and desirable. The standard harmonic oscillator, rigid rotor estimate (solid blue line in Figure 4) yields smaller magnitudes for $\Delta U_{\text {ads }}$ than the coupled, anharmonic RBPIMC values, with the difference increasing with temperature. Despite this qualitative disagreement, it should be noted that, at all temperatures shown in Figure 4, the discrepancy is less than 1 kcal.mol ${ }^{-1}$ $\left(4.2 \mathrm{~kJ} \mathrm{~mol}^{-1}\right)$ chemical accuracy. This suggests that the quantitative description of $\mathrm{H}_{2}$ adsorption requires significantly better than chemical accuracy.

One of the most common methods used in the literature to improve harmonic estimates of internal energy, enthalpy and entropy is the scaling of harmonic vibrational frequencies. ${ }^{15}$ Scaling factors correct for anharmonicity, coupling, incomplete incorporation of electron correlation and the use of finite, one-electron basis sets. They have been derived from comparison of calculated frequencies with accurate experimental results for a range of gas-

phase species. Applying the appropriate literature M05-2X/6-311+G(2df,p) scaling factors ${ }^{15}$ will only very slightly lower the harmonic estimates for $\Delta U_{\text {ads }}$ (and $\Delta H_{\text {ads }}$ ); the strength of the binding interaction is still significantly underestimated. The application of such scaling factors assumes transferability from the test set to a weakly bound complex, such as $\mathrm{H}_{2^{-}}$ $\mathrm{Li}^{+}$-benzene, and further assumes that the relevant degrees of freedom can be described as vibrations. Neither of these assumptions are likely to be true for $\mathrm{H}_{2}-\mathrm{Li}^{+}$-benzene. Rather than present scaled results we have chosen to explicitly model the effects of anharmonicity and coupling, only two of the contributions to literature scaling factors.

Our previous RBDMC simulations identified the most anharmonic intramolecular modes 
in $\mathrm{H}_{2}-\mathrm{Li}^{+}$-benzene as the helicopter and ferris wheel $\mathrm{H}_{2}$ rotations. ${ }^{20}$ We found that treating these modes as one-dimensional free and hindered rotors, respectively, rather than as harmonic oscillators, significantly improved the calculated ZPE of this species. ${ }^{20}$

The red, dashed line in Figure 4 represents $\Delta U_{\text {ads }}$ calculated with all vibrational modes treated as harmonic oscillators, except for the helicopter and ferris wheel $\mathrm{H}_{2}$ rotations, which were treated as free and hindered rotors, respectively. These simple corrections dramatically improve the harmonic oscillator, rigid rotor result, yielding $\Delta U_{\text {ads }}$ within $0.4 \mathrm{~kJ} . \mathrm{mol}^{-1}$ of the 1D DVR result over the temperature range $0 \mathrm{~K}$ to $300 \mathrm{~K}$. This simple approximation corrects for the majority of the anharmonicity in $\Delta U_{\text {ads }}$. Treating the $\mathrm{H}_{2}$ helicopter and ferris wheel rotations as free and hindered rotors, however, over-corrects the $\Delta U_{\text {ads }}$ at $0 \mathrm{~K}$, with respect to the 1D DVR value, but under-corrects $\Delta U_{\text {ads }}$ for temperatures greater than approximately $77 \mathrm{~K}$. Assuming the $\mathrm{H}_{2}$ helicopter rotation at $0 \mathrm{~K}$ behaves as a free rotor underestimates the one-dimensional ZPE in this mode; there is no ZPE for a free rotor whereas the "real" onedimensional mode has very small energetic barriers to rotation. As temperature increases, this becomes less important as the rotational energy in the helicopter mode becomes larger. It is also worth noting that the $\mathrm{H}_{2}$ ferris wheel hindered rotation has relatively widely spaced energy levels such that excited states of this mode are largely unpopulated even at $300 \mathrm{~K}$. As temperature increases the red, long-dashed line in Figure 4 increasingly deviates from the 1D DVR estimate of $\Delta U_{\text {ads }}$, suggesting that there are other vibrational modes in the system that are anharmonic. In this case the only other vibrational modes exhibiting any significant degree of anharmonicity are the two orthogonal $\mathrm{H}_{2}$ rocking modes in $\theta$. These are low frequency modes and their vibrational excited states are populated at relatively low temperatures; thermal energy is dominated by the lowest frequency modes. As temperature increases, a two-dimensional anharmonic treatment of these modes will improve the predicted $\Delta U_{\text {ads }}$. These four modes: the $\mathrm{H}_{2}$ helicopter and ferris wheel free and hindered rotations and the two orthogonal $\mathrm{H}_{2}$ rocking modes are characteristic of physisorbed $\mathrm{H}_{2}$. The simple corrections we suggest are therefore likely to be of value in any study of $\mathrm{H}_{2}$ adsorption.

\section{Adsorption Enthalpy, $\Delta H_{a d s}$}

The adsorption enthalpy, $\Delta H_{\mathrm{ads}}=\Delta U_{\mathrm{ads}}+p V$, for $\mathrm{H}_{2}$ adsorption onto $\mathrm{Li}^{+}$-benzene, is plotted in Figure 5 as a function of temperature. Within the ideal gas approximation, $\Delta H_{\text {ads }}$ 
$=\Delta U_{\mathrm{ads}}-k_{B} T$. Various estimates of $\Delta H_{\mathrm{ads}}$ are shown in Figure 5 , with the same key as shown in Figure 4.

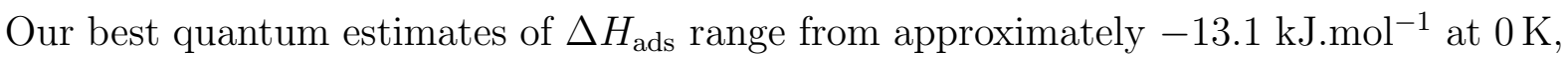

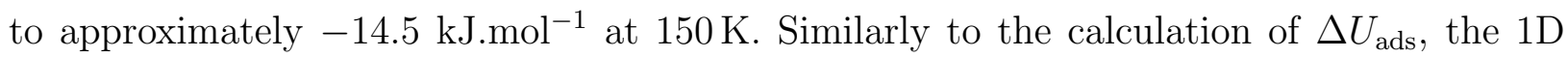
DVR estimate yields slightly smaller magnitudes for $\Delta H_{\text {ads }}$ than the RBDMC and RBPIMC estimates, with coupling accounting for between 1.1 and $0.7 \mathrm{~kJ} \cdot \mathrm{mol}^{-1}$ between $0 \mathrm{~K}$ and $150 \mathrm{~K}$. Again, anharmonicity is dominated by the $\mathrm{H}_{2}$ helicopter and ferris wheel rotations. The estimates obtained treating these modes as a free rotor and one-dimensional hindered rotor (red, dashed line in Figure 5) are within $0.4 \mathrm{~kJ} \cdot \mathrm{mol}^{-1}$ of the $1 \mathrm{D}$ DVR result over the temperature range shown in Figure 5. Importantly, the largest magnitudes of $\Delta H_{\text {ads }}$ were calculated from the RBPIMC simulations at the highest temperature, $150 \mathrm{~K}$, and the 1D DVR results gives the largest magnitude of $\Delta H_{\text {ads }}$ at approximately $250 \mathrm{~K}$.

Although the classical $\Delta H_{\text {ads }}$ is more exothermic than the quantum estimates, in contrast to the quantum results, the classical $\Delta H_{\text {ads }}$ increases monotonically with increasing temperature. This can be seen clearly in the equipartion theorem classical estimate (light green alternating long- and short-dashed line in Figure 5) which has a slope of $k_{B} T$. Similarly to Figure 4, the equipartition result provides an excellent approximation to the fully coupled, anharmonic classical RBPIMC simulations.

The results shown in Figure 5 suggest that $\mathrm{H}_{2}$ adsorption enthalpies calculated from classical simulations should be treated with caution. They are likely to both overestimate the $\mathrm{H}_{2}$ binding interaction and show an incorrect temperature dependence.

Experimental enthalpies of adsorption of $\mathrm{H}_{2}$ to MOF materials with bare metal sites are typically in the range of -8 to $-12 \mathrm{~kJ} \mathrm{~mol}^{-1},{ }^{22}$ placing the adsorption enthalpy calculated here at least $2 \mathrm{~kJ} \cdot \mathrm{mol}^{-1}$ larger in magnitude than experiment. This can be attributed, at least in part, to the differences between our model system and a real bare-metal site in a MOF material. The effective positive charge on a bare metal site in a MOF material can be significantly reduced by the bonding interactions of the metal center with the organic linkers. The effective charge on the $\mathrm{Li}^{+}$ion in $\mathrm{Li}^{+}$-benzene, however, is very large, only $30 \%$ of the +1 positive charge is "lost" to benzene. ${ }^{16}$ Although other computational simulations of Li-doped MOF materials also show large localised positive charge on the lithium, in some cases larger than the +0.7 e found for $\mathrm{H}_{2}-\mathrm{Li}^{+}$-benzene, ${ }^{50}$ we suggest that our $\Delta H_{\text {ads }}=-14.5$ $\mathrm{kJ} . \mathrm{mol}^{-1}$ be considered a lower bound to the enthalpy of adsorption of $\mathrm{H}_{2}$ to a Li-doped 
MOF or carbon-based material.

\section{SUMMARY AND CONCLUDING REMARKS}

We have investigated the nature of adsorption of $\mathrm{H}_{2}$ to $\mathrm{Li}^{+}$-benzene at finite temperatures using RBPIMC simulations on an eight-dimensional interpolated PES constructed from M05-2X/6-311+G(2df,p) density functional theory calculations. Classical results were obtained from $P=1$ RBPIMC simulations and quantum results were obtained from $P \rightarrow \infty$ simulations.

We investigated the temperature dependence of quantum effects by examining the temperature dependence of both quantum and classical probability density histograms describing the adsorbed $\mathrm{H}_{2}$. Quantum effects broaden the probability densities of the $\mathrm{H}_{2}$ center-ofmass to $\mathrm{Li}^{+}$distance, the $\theta$ polar coordinate and the "ferris" wheel rotation coordinate. The quantum probability density histograms show less temperature dependence than their classical analogues.

Our reported harmonic oscillator, rigid rotor, 1D DVR, and 8-dimensional quantum RBPIMC calculations provide successively more accurate estimates for the change in internal energy, $\Delta U_{\text {ads }}$, for $\mathrm{H}_{2}$ adsorption onto $\mathrm{Li}^{+}$-benzene, as a function of temperature. Applying an ideal gas correction enables us to obtain a similar hierarchy of estimates for the enthalpy of adsorption, $\Delta H_{\text {ads }}$. These estimates indicate that quantum effects are important in this system, even at room temperature, and must be included in any accurate study of $\mathrm{H}_{2}$ adsorption. They also show that anharmonicity is more important in the calculation of $U$ and $H$ than coupling; the 1D DVR results agree well with the higher dimensionality RBPIMC results. As the effort to undertake these calculations increases with dimensionality, however, we have described simple corrections to the standard harmonic oscillator, rigid rotor thermochemical expressions for $U$ and $H$ which encapsulate the majority of the anharmonicity in the $\mathrm{H}_{2}-\mathrm{Li}^{+}$-benzene system. Approximating the $\mathrm{H}_{2}$ helicopter and ferris wheel rotations as free and hindered rotors, respectively, allow a significantly more accurate calculation of internal energy. Moreover, these simple corrections are applicable to any simulation of hydrogen adsorption.

Overall our calculations suggest that classical simulations of $\mathrm{H}_{2}$ adsorption should be treated with caution. Our classical results give significantly more favourable $\mathrm{H}_{2}$ binding 
interactions than the quantum simulations, and classical thermochemical properties exhibit an incorrect temperature dependence, with respect to the quantum equivalents.

\section{ACKNOWLEDGMENTS}

This work was supported by the Australian Research Council (DP0665065) and the University of Sydney (Bridging Support Grant 2012-00076). L. P. L., S. J. K. and J. H. D. acknowledge the financial support of an Australian Postgraduate Award. Parts of this research were undertaken on the NCI National Computational Infrastructure Facility in Canberra, Australia, which is supported by the Australian Commonwealth Government.

\section{REFERENCES}

${ }^{1}$ D. Zhao, D. Yuan, and H.-C. Zhou, Energy Environ. Sci. 1, 222 (2008).

${ }^{2}$ P. Bénard, R. Chahine, P. A. Chandonia, D. Cossement, G. Dorval-Douville, L. Lafi, P. Lachance, R. Paggiaro, and E. Poirier, J. Alloys Compd. 446-447, 380 (2007).

${ }^{3}$ Fuel Cell Technologies Office Multi-Year Research, Development and Demonstration Plan (U.S. Department of Energy, Washington, DC, 2012) p 3.3.

${ }^{4}$ Y. Li and R. T. Yang, Langmuir 23, 12937 (2007).

${ }^{5}$ A. G. Wong-Foy, A. J. Matzger, and O. M. Yaghi, J. Am. Chem. Soc. 128, 3494 (2006).

${ }^{6}$ H. Furukawa, M. A. Miller, and O. M. Yaghi, J. Mater. Chem. 17, 3197 (2007).

${ }^{7}$ K. M. Thomas, Catal. Today 120, 389 (2007).

${ }^{8}$ S. Bordiga, J. G. Vitillo, G. Ricchiardi, L. Regli, D. Cocina, A. Zecchina, B. Arstad, M. Bjørgen, J. Hafizovic, and K. P. Lillerud, J. Phys. Chem. B 109, 18237 (2005).

${ }^{9}$ F. Mulder, T. Dingemans, H. Schimmel, A. Ramirez-Cuesta, and G. Kearley, Chem. Phys. 351, 72 (2008).

${ }^{10}$ S. K. Bhatia and A. L. Myers, Langmuir 22, 1688 (2006).

${ }^{11}$ R. C. Lochan and M. Head-Gordon, Phys. Chem. Chem. Phys. 8, 1357 (2006).

${ }^{12}$ L. J. Murray, M. Dincă, and J. R. Long, Chem. Soc. Rev. 38, 1294 (2009).

${ }^{13}$ M. Dincǎ and J. R. Long, Angew. Chem. Int. Ed. 47, 6766 (2008).

${ }^{14}$ S. M. Chavan, O. Zavorotynska, C. Lamberti, and S. Bordiga, Dalton Trans. 42, 12586 (2013). 
${ }^{15}$ J. P. Merrick, D. Moran, and L. Radom, J. Phys. Chem. A 111, 11683 (2007).

${ }^{16}$ S. J. Kolmann, B. Chan, and M. J. Jordan, Chem. Phys. Lett. 467, 126 (2008).

${ }^{17}$ A. Lovell, F. Fernandez-Alonso, N. T. Skipper, K. Refson, S. M. Bennington, and S. F. Parker, Phys. Rev. Lett. 101, 126101 (2008).

${ }^{18}$ M. Barbatti, G. Jalbert, and M. A. C. Nascimento, J. Chem. Phys. 114, 2213 (2001).

${ }^{19}$ S. A. FitzGerald, K. Allen, P. Landerman, J. Hopkins, J. Matters, R. Myers, and J. L. C. Rowsell, Phys. Rev. B 77, 224301 (2008).

${ }^{20}$ S. J. Kolmann, J. H. D’Arcy, and M. J. T. Jordan, J. Chem. Phys. 139, 234305 (2013).

${ }^{21}$ H. J. Park, D.-W. Lim, W. S. Yang, T.-R. Oh, and M. P. Suh, Chem.-Eur. J. 17, 7251 (2011).

${ }^{22}$ M. P. Suh, H. J. Park, T. K. Prasad, and D.-W. Lim, Chem. Rev. 112, 782 (2012).

${ }^{23}$ R. Feynman and A. Hibbs, Quantum Mechanics and Path Integrals: Emended Edition (Dover Publications, Incorporated, 2012).

${ }^{24}$ R. P. Feynman, Statistical Mechanics (Benjamin, New York, 1972).

${ }^{25}$ GROW 2.2, available from the authors. Contact m.jordan@chem.usyd.edu.au.

${ }^{26}$ Y. Zhao, N. E. Schultz, and D. G. Truhlar, J. Chem. Theory Comput. 2, 364 (2006).

${ }^{27}$ T. Clark, J. Chandrasekhar, G. W. Spitznagel, and P. von Ragué Schleyer, J. Comput. Chem. 4, 294 (1983).

${ }^{28}$ R. Krishnan, J. S. Binkley, R. Seeger, and J. A. Pople, J. Chem. Phys. 72, 650 (1980).

${ }^{29}$ M. J. Frisch, J. A. Pople, and J. S. Binkley, J. Chem. Phys. 80, 3265 (1984).

${ }^{30}$ M. J. Frisch, G. W. Trucks, H. B. Schlegel, G. E. Scuseria, M. A. Robb, J. R. Cheeseman, J. A. Montgomery, Jr., T. Vreven, K. N. Kudin, J. C. Burant, J. M. Millam, S. S. Iyengar, J. Tomasi, V. Barone, B. Mennucci, M. Cossi, G. Scalmani, N. Rega, G. A. Petersson, H. Nakatsuji, M. Hada, M. Ehara, K. Toyota, R. Fukuda, J. Hasegawa, M. Ishida, T. Nakajima, Y. Honda, O. Kitao, H. Nakai, M. Klene, X. Li, J. E. Knox, H. P. Hratchian, J. B. Cross, V. Bakken, C. Adamo, J. Jaramillo, R. Gomperts, R. E. Stratmann, O. Yazyev, A. J. Austin, R. Cammi, C. Pomelli, J. W. Ochterski, P. Y. Ayala, K. Morokuma, G. A. Voth, P. Salvador, J. J. Dannenberg, V. G. Zakrzewski, S. Dapprich, A. D. Daniels, M. C. Strain, O. Farkas, D. K. Malick, A. D. Rabuck, K. Raghavachari, J. B. Foresman, J. V. Ortiz, Q. Cui, A. G. Baboul, S. Clifford, J. Cioslowski, B. B. Stefanov, G. Liu, A. Liashenko, P. Piskorz, I. Komaromi, R. L. Martin, D. J. Fox, T. Keith, M. A. Al-Laham, C. Y. Peng, A. Nanayakkara, M. Challacombe, P. M. W. Gill, B. Johnson, W. Chen, M. W. Wong, 
C. Gonzalez, and J. A. Pople, Gaussian 03, Revision E.01, Gaussian, Inc., Wallingford, CT 2004.

${ }^{31}$ M. J. Frisch, G. W. Trucks, H. B. Schlegel, G. E. Scuseria, M. A. Robb, J. R. Cheeseman, G. Scalmani, V. Barone, B. Mennucci, G. A. Petersson, H. Nakatsuji, M. Caricato, X. Li, H. P. Hratchian, A. F. Izmaylov, J. Bloino, G. Zheng, J. L. Sonnenberg, M. Hada, M. Ehara, K. Toyota, R. Fukuda, J. Hasegawa, M. Ishida, T. Nakajima, Y. Honda, O. Kitao, H. Nakai, T. Vreven, J. A. Montgomery, Jr., J. E. Peralta, F. Ogliaro, M. Bearpark, J. J. Heyd, E. Brothers, K. N. Kudin, V. N. Staroverov, R. Kobayashi, J. Normand, K. Raghavachari, A. Rendell, J. C. Burant, S. S. Iyengar, J. Tomasi, M. Cossi, N. Rega, J. M. Millam, M. Klene, J. E. Knox, J. B. Cross, V. Bakken, C. Adamo, J. Jaramillo, R. Gomperts, R. E. Stratmann, O. Yazyev, A. J. Austin, R. Cammi, C. Pomelli, J. W. Ochterski, R. L. Martin, K. Morokuma, V. G. Zakrzewski, G. A. Voth, P. Salvador, J. J. Dannenberg, S. Dapprich, A. D. Daniels, O. Farkas, J. B. Foresman, J. V. Ortiz, J. Cioslowski, and D. J. Fox, Gaussian 09 Revision D.01, gaussian Inc. Wallingford, CT 2009

${ }^{32}$ M. A. Collins, Theor. Chem. Acc. 108, 313 (2002).

${ }^{33}$ M. J. T. Jordan, K. C. Thompson, and M. A. Collins, J. Chem. Phys. 102, 5647 (1995).

${ }^{34}$ K. C. Thompson, M. J. T. Jordan, and M. A. Collins, J. Chem. Phys. 108, 8302 (1998).

${ }^{35}$ R. P. A. Bettens and M. A. Collins, J. Chem. Phys. 111, 816 (1999).

${ }^{36}$ S. J. Kolmann and M. J. T. Jordan, J. Chem. Phys. 132, 054105 (2010).

${ }^{37}$ B. J. Berne and D. Thirumalai, Annu. Rev. Phys. Chem. 37, 401 (1986).

${ }^{38}$ D. Ceperley, Rev. Mod. Phys. 67, 279 (1995).

${ }^{39}$ M. E. Tuckerman, Statistical Mechanics: Theory and Molecular Simulation (Oxford University Press, New York, 2010).

${ }^{40}$ See supplemental material at [URL will be inserted by AIP] for a consistent derivation of the rigid body path integral formalism and staging algorithm used in this work and for an analysis of the parameters required for convergence of the internal energy at the various temperatures considered.

${ }^{41}$ H. F. Trotter, Proc. Am. Math. Soc. 10, 545 (1959).

${ }^{42}$ M. H. Müser and B. J. Berne, Phys. Rev. Lett. 77, 2638 (1996).

${ }^{43}$ M. H. Müser, Molecular Simulation 17, 131 (1996).

${ }^{44}$ E. G. Noya, L. M. Sesé, R. Ramírez, C. McBride, M. M. Conde, and C. Vega, Mol. Phys. 
109, 149 (2011).

${ }^{45}$ C. McBride, E. G. Noya, and C. Vega, Comp. Phys. Comm. 184, 885 (2013).

${ }^{46}$ E. L. Pollock and D. M. Ceperley, Phys. Rev. B 39, 2555 (1984).

${ }^{47}$ H. Flyvbjerg and H. G. Petersen, J. Chem. Phys. 91, 461 (1989).

${ }^{48}$ D. McQuarrie, Statistical Mechanics (University Science Books, 2000).

${ }^{49}$ D. T. Colbert and W. H. Miller, J. Chem. Phys. 96, 1982 (1992).

${ }^{50}$ A. Blomqvist, C. M. Araújo, P. Srepusharawoot, and R. Ahuja, Proc. Natl. Acad. Sci. USA 104, 20173 (2007). 


\section{TABLES}

TABLE I. Expectation values, $\langle A\rangle$, and full-width-half-maxima, $A_{\mathrm{FWHM}}$, of the probability density histograms for the $r(\AA)$ and $\theta\left(^{\circ}\right)$ coordinates (Figure 2), as a function of Temperature $(\mathrm{K})$, from classical ("c"; $P=1$ ) and quantum ("q"; $P \rightarrow \infty$ ) RBPIMC simulations.

\begin{tabular}{lllllllll}
\hline \hline Temp. & $\langle r\rangle_{c}$ & $\langle r\rangle_{q}$ & $r_{F W H M, c}$ & $r_{F W H M, q}$ & $\langle\theta\rangle_{c}$ & $\langle\theta\rangle_{q}$ & $\theta_{F W H M, c}$ & $\theta_{F W H M, q}$ \\
\hline 0 & & 2.154 & & & & 18.4 & & \\
77 & 2.069 & 2.168 & 0.203 & 0.413 & 23.6 & 25.5 & 29.1 & 32.4 \\
90 & 2.077 & 2.172 & 0.222 & 0.416 & 24.8 & 26.5 & 30.8 & 33.5 \\
100 & 2.083 & 2.175 & 0.236 & 0.420 & 25.6 & 27.2 & 31.9 & 34.0 \\
110 & 2.090 & 2.179 & 0.250 & 0.423 & 26.3 & 27.8 & 33.0 & 34.8 \\
120 & 2.096 & 2.184 & 0.265 & 0.427 & 27.0 & 28.4 & 33.9 & 35.4 \\
130 & 2.103 & 2.189 & 0.278 & 0.432 & 27.7 & 29.0 & 34.6 & 35.8 \\
140 & 2.111 & 2.199 & 0.291 & 0.438 & 28.3 & 29.6 & 35.3 & 36.4 \\
150 & 2.119 & 2.205 & 0.303 & 0.444 & 28.9 & 30.2 & 36.0 & 36.9 \\
\hline \hline
\end{tabular}

TABLE II. Full-width-half-maxima $\left(^{\circ}\right)$ of the "ferris wheel" probability density histograms (Figure 3) as a function of Temperature (K) from the classical and quantum RBPIMC simulations.

\begin{tabular}{ccc}
\hline \hline Temp. & Classical $(P=1)$ & Quantum $(P \rightarrow \infty)$ \\
\hline 77 & 17.3 & 43.9 \\
90 & 18.7 & 44.1 \\
100 & 19.8 & 44.2 \\
110 & 20.7 & 44.2 \\
120 & 21.8 & 44.3 \\
130 & 22.7 & 44.2 \\
140 & 23.6 & 44.5 \\
150 & 24.5 & 44.4 \\
\hline \hline
\end{tabular}




\section{FIGURE CAPTIONS}

FIG. 1. The polar coordinate system, $\{r, \theta, \phi\}$, used to describe $\mathrm{H}_{2}-\mathrm{Li}^{+}$-benzene. Hydrogen atoms are coloured white, carbon gray and lithium pink.

FIG. 2. Selected nuclear probability density histograms in the (a) $r$ and (b) the $\theta$ coordinate from RBDMC and RBPIMC simulations. $0 \mathrm{~K}$, black (RBDMC); $77 \mathrm{~K}$ (red), $100 \mathrm{~K}$ (green), $120 \mathrm{~K}$ (blue) and $150 \mathrm{~K}$ (orange) from RBPIMC simulations. Quantum results are shown as solid lines and classical results as dashed lines. Errors, representing twice the standard error of the mean of 10 independent RBDMC simulations are shown as grey shading around the RBDMC line. RBPIMC errors are within the thickness of the lines.

FIG. 3. Selected nuclear probability density histograms in the $\mathrm{H}_{2}$ "ferris wheel" angle from RBPIMC or RBDMC simulations. 0 K, black (RBDMC); $77 \mathrm{~K}$ (red), $100 \mathrm{~K}$ (green), $120 \mathrm{~K}$ (blue) and $150 \mathrm{~K}$ (orange) from RBPIMC simulations. Quantum results are shown as solid lines and classical results as dashed lines. Errors, representing twice the standard error of the mean of 10 independent RBDMC simulations are shown as grey shading around the RBDMC line. RBPIMC errors are within the thickness of the lines.

FIG. 4. Estimates, as indicated, of the change in internal energy upon adsorption, $\Delta U_{\text {ads }}$, for $\mathrm{H}_{2}$ adsorption onto $\mathrm{Li}^{+}$-benzene, as a function of temperature. Errors in the classical RBPIMC results are within the size of the symbols used.

FIG. 5. Estimates, as indicated, of the enthalpy of adsorption, $\Delta H_{\text {ads }}$, for $\mathrm{H}_{2}$ adsorption onto $\mathrm{Li}^{+}$-benzene, as a function of temperature. 